\section{QUANTUM SOURCES}

\section{Dual wavelength chip}

Nat. Phys. https://doi.org/c2cz (2019)

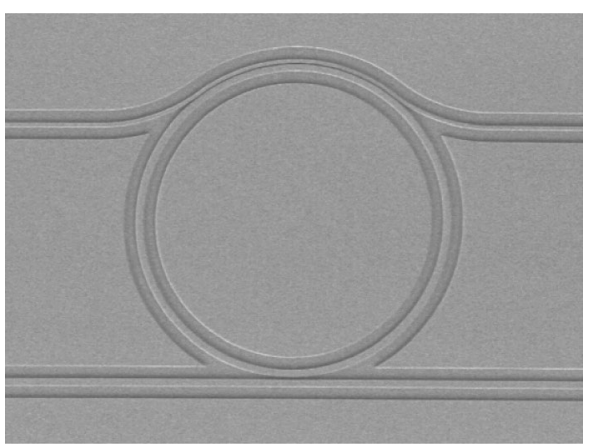

Credit: Springer Nature Ltd

Long-distance fibre-based quantum communications systems tend to operate in the telecommunications wavelength band $(1.5 \mu \mathrm{m})$ in the near-infrared (NIR). In contrast, quantum systems for performing local operations, such as storage and computation, make use of optical transitions in the visible or shorter NIR wavelengths from trapped ions or atoms, defect centres, quantum dots and spins in a crystal. To link these two systems together entangled photon pair sources that bridge the telecoms and visible bands are desired. Using a high- $Q$ silicon nitride microresonator platform, Xiyuan Lu and co-workers from the USA have now demonstrated a system that generates narrow-band visible-telecom photon pairs with a coincidence-toaccidental ratio of up to $3,780 \pm 140$ at an on-chip pair flux of $1,200 \pm 300$ pairs per second, at sub-milliwatt pump power. Unlike existing visible-telecom pair sources, the system combines an on-chip solution with phase- and frequency-matching of narrowband modes over a spectral separation of $>250 \mathrm{THz}$. The team report time-energy entanglement of the pairs with a visibility of $82.7 \pm 0.2 \%$. When the entanglement was distributed over $>20 \mathrm{~km}$ in optical fibre, a visibility of $58 \pm 1 \%$ was measured. $R W$

https://doi.org/10.1038/s41566-019-0386-6

\section{OPTICAL FORCE}

\section{Dimer nanomotors}

Light Sci. Appl. 7, 105 (2018)

Recent simulations predict that dissimilar particle dimers can experience a net force that is transverse to the direction of the illuminating light and may be exploited for optically driven nanomotor applications. Now, Yuval Yifat and colleagues in Chicago have directly confirmed the effect and have measured the net forces in asymmetric nanoparticle dimers excited by plane waves. A continuous-wave Ti:sapphire laser (790 nm wavelength) illuminated a mixture of $\sim 150$-nm-diameter and $\sim 180$-nm-diameter Ag nanoparticles in a $3.4-\mu \mathrm{m}$-radius optical ring-trap and the positions of the particles were tracked. Both homodimer and heterodimer pairs were investigated. The anticipated optically driven motion was confirmed for heterodimers that were sufficiently close for optical coupling to occur (separations less than $1.2 \mu \mathrm{m}$ ) and is attributed to an asymmetric scattering pattern. The team hopes that asymmetric nanoparticle assemblies can actually be used as active colloids and optically driven 'nanoswimmers' that may be useful in soft condensed-matter and biophysics research.

\section{METAMATERIALS \\ One-way chiral light}

Science 363, 148-151 (2019)

Weyl points are singular points of degeneracy in three-dimensional reciprocal (momentum) space at which two energy bands intersect. Such points were experimentally demonstrated in the photonic domain in 2015 and growing interest and applications are now abounding. However, while the fact that Weyl points carry topological charges defined by the Chern numbers has been exploited in other fields of physics, photonics is still lagging. Hongwei Jia and a team from the UK, China and the US have now experimentally observed microwave zero-order Landau levels exhibiting one-way chiral propagation. They achieved this by making designer unit cells of metamaterials that generate an effective gauge field (that is, artificial magnetic field), which was confirmed experimentally and by numerical simulations. Apart from the fundamental nature of the demonstration, the concept, which does not rely on time-reversal symmetry breaking, may allow for means of robust photonic transport that could be useful for information processing and other applications.

DFPP

https://doi.org/10.1038/s41566-019-0382-x

\section{OPTICAL MEMORY}

\section{Terahertz read-out}

Appl. Phys. Lett. 114, 011105 (2019)

A multilevel optical memory that encodes information as the strength of transmission of terahertz radiation through an indium oxide nanoparticle film has been fabricated by scientists in Beijing, China. Hongyu Ji and co-workers from Capital Normal University and the Institution of Semiconductors dissolved 10-nm-diameter indium oxide nanoparticles in ethanol and then spin-coated the mixture onto a quartz substrate and annealed it at $340{ }^{\circ} \mathrm{C}$ for 1 hour. Experiments indicate that the terahertz transmission of the sample could be strongly modified by exposure to short-wavelength (ultraviolet or blue) light. The drop in the transmission of the sample was shown to relate to the intensity of the light exposure. The team used four relative transmission levels of 1.0, $0.8,0.6$ and 0.4 to encode information into the sample. If the sample was stored in an oxygenrich environment, such as air, the memory effect was shown to decay and disappear after the light exposure stopped, allowing information to be wiped. However, when stored in nitrogen, the memory became a stable, non-volatile device. 